\title{
Radio and X-ray nebulae associated with PSR J1509-5850
}

\author{
C. Y. Hui and W. Becker
}

\begin{abstract}
Max-Planck Institut für Extraterrestrische Physik, Giessenbachstrasse 1, 85741 Garching bei München, Germany
e-mail: dhui@mpe.mpg.de
\end{abstract}

Received 10 April 2007 / Accepted 10 May 2007

\begin{abstract}
We have discovered a long radio trail at $843 \mathrm{MHz}$ which is apparently associated with middle-age pulsar PSR J1509-5850. The radio trail has a length of $\sim 7$ arcmin. In X-rays, Chandra observation of PSR J1509-5850 reveals an associated X-ray trail which extends in the same orientation as the radio trail. Moreover, two clumpy structures are observed along the radio trail. The larger one is proposed to be the supernova remnant (SNR) candidate MSC 319.9-0.7. Faint X-ray enhancement at the position of the SNR candidate is found in the Chandra data.
\end{abstract}

Key words. pulsars: individual: PSR J1509-5850 - stars: neutron - X-rays: stars

\section{Introduction}

It is generally believed that a significant fraction of the rotational energy of a pulsar leaves the magnetosphere in the form of a magnetized pulsar wind consisting of electromagnetic radiation and high energy particles. In view of this, it is important energetically to study the physical properties of this wind. When the relativistic wind particles interact with the shocked interstellar medium, the charged particles will be accelerated in the shock and hence synchrotron radiation from radio to $\mathrm{X}$-ray is generated. To obtain a better understanding of the interaction nature, multiwavelength studies of the pulsar wind nebulae are of prime importance. X-ray and radio observations have recently revealed a number of pulsar wind nebulae. However, very few shocked emissions are detected in both the X-ray and radio regimes (cf. see Hui \& Becker 2006, and references therein).

PSR J1509-5850 was discovered by Manchester et al. (2001) in the Parkes Multibeam Pulsar Survey. The pulsar has a rotation period of $P=88.9 \mathrm{~ms}$ and a period derivative of $\dot{P}=9.17 \times 10^{-15} \mathrm{~s} \mathrm{~s}^{-1}$. These spin parameters imply a characteristic age of $1.54 \times 10^{5}$ yrs, a dipole surface magnetic field of $B_{\perp}=9.14 \times 10^{11} \mathrm{G}$ and a spin-down luminosity of $5.1 \times 10^{35} \mathrm{erg} \mathrm{s}^{-1}$ (cf. Table 1). The radio dispersion measure yields a distance of approximately $3.81 \mathrm{kpc}$ based on the galactic free electron model of Taylor \& Cordes (1993). Using the model of Cordes \& Lazio (2002) the dispersion measure based distance is estimated to be $2.56 \mathrm{kpc}$. The proper motion of this pulsar is not yet known. Recently, a brief X-ray study of the field of PSR J1509-5850 was presented by Kargaltsev et al. (2006). The authors reported that a trail-like pulsar wind nebula associated with PSR J1509-5850 was observed in a Chandra observation. The X-ray nebula was found to be extended in the south-west direction.

In this paper we report the discovery of a possible radio counterpart of the X-ray trail associated with PSR J1509-5850 and provide a detailed X-ray analysis of the trail. In Sect. 2 we describe the observations and the data analysis and in Sect. 3 we summarize and discuss our results.
Table 1. Pulsar parameters of PSR J1509-5850 (from Manchester et al. 2005).

\begin{tabular}{lc}
\hline \hline & \\
\hline Right Ascension (J2000) & $15^{\mathrm{h}} 09^{\mathrm{m}} 27.13^{\mathrm{s}}$ \\
Declination (J2000) & $-58^{\circ} 50^{\prime} 56.1^{\prime \prime}$ \\
Pulsar Period, $P(\mathrm{~s})$ & 0.088921760489 \\
Period derivative $\dot{P}\left(10^{-15} \mathrm{~s} \mathrm{~s}^{-1}\right)$ & 9.1698 \\
Age $\left(10^{5}\right.$ yrs) & 1.54 \\
Surface dipole magnetic field $\left(10^{12} \mathrm{G}\right)$ & 0.914 \\
Epoch of Period (MJD) & 51463 \\
Dispersion Measure $\left(\mathrm{pc} \mathrm{cm}^{-3}\right)$ & 137.7 \\
Dispersion based distance $(\mathrm{kpc})$ & $\sim 2.6-3.8$ \\
Spin-down Luminosity $\left(10^{35}\right) \mathrm{erg} \mathrm{s}^{-1}$ & 5.1 \\
\hline
\end{tabular}

\section{Observations and data analysis}

PSR J1509-5850 was observed with Chandra in 2003 February 9-10 (Obs ID: 3513) with the Advanced CCD Imaging Spectrometer (ACIS). The pulsar is located on the backilluminated (BI) ACIS-S3 chip which has superior quantum efficiency within the spectroscopic array. Standard processed level2 data were used. The effective exposure is approximately $40 \mathrm{ks}$.

Chandra observation revealed an X-ray trail associated with PSR J1509-5850. The signal-to-noise ratios for the pulsar and the trail were $\sim 19$ and $\sim 3$ in $0.5-8 \mathrm{keV}$ respectively. The X-ray image of the $4 \times 4$ arcmin field near to PSR J1509-5850 is shown in Fig. 1. The binning factor of the image is 0.5 arcsec. Adaptive smoothing with a Gaussian kernel of $\sigma<3$ arcsec has been applied to the image. The trail appears to have a length of $\sim 2$ arcmin. From a Digitized Sky Survey (DSS) image, 25 bright field stars are found in the field of view of Fig. 1. We subsequently identified the magnitudes of these stars from the USNOA2.0 catalog (Monet et al. 1998), which are within the range of $B \sim 10-18$. Their positions are plotted as white circles in Fig. 1.

For the spectral analysis, we extracted the spectrum of PSR J1509-5850 from a circle of 4 arcsec radius (encircled energy $\sim 99 \%$ ) centered on the pulsar position. To minimize the 


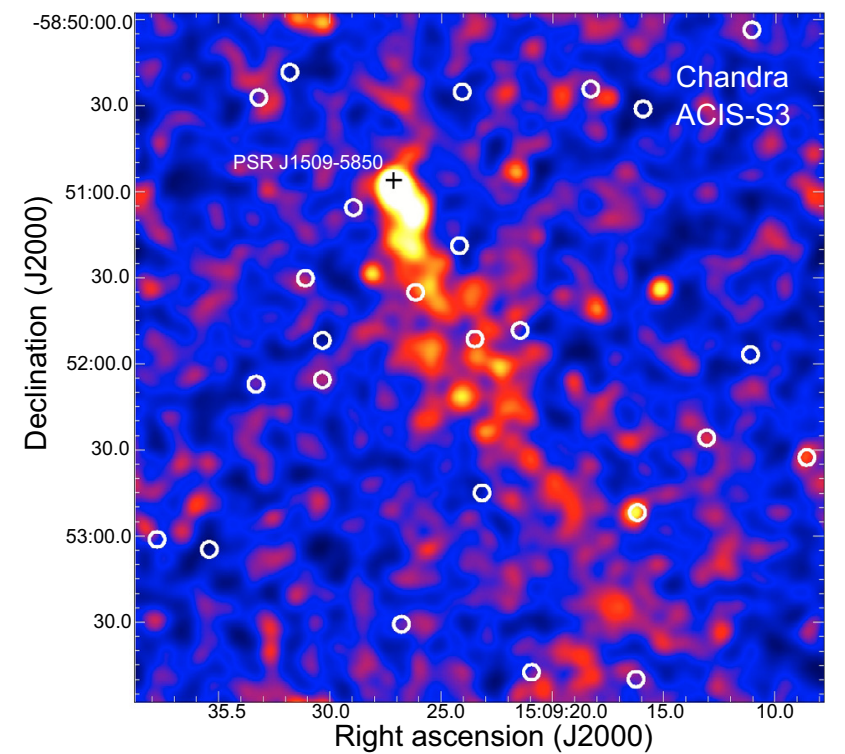

Fig. 1. Chandra's $4 \times 4$ arcmin view of PSR J1509-5850 and X-ray trail in the energy band $0.3-8 \mathrm{keV}$. Pulsar position is indicated by the black cross. White circles indicate the positions of field stars identified in the DSS image.

possible contamination from field stars, the spectrum from the trail was extracted within a box of $25 \times 95$ arcsec, oriented along the direction of the trail emission. Even with this consideration, there are two stars with magnitude $B=17$ and $B=16.4$ located on the trail (cf. Fig. 1) and lie unavoidably in the extraction region. Without the knowledge of the extinctions, we are not able to estimate the possible contribution in X-ray from these two stars. The background spectra were extracted from the low count regions nearby. After background subtraction, there are $\sim 100$ net counts and $\sim 270$ net counts extracted from the pulsar and the trail in $0.5-8 \mathrm{keV}$ respectively. Response files were computed with CIAO tools MKRMF and MKARF. Spectra were dynamically binned to have at least 10 counts per bin for the pulsar and 30 counts per bin for the trail. All spectral fittings were performed in the energy range of $0.5-8 \mathrm{keV}$ using XSPEC 11.3.1. The degradation of the ACIS quantum efficiency was corrected by XSPEC model ACISABS. All quoted errors are $1-\sigma$ and were computed for 1 parameter in interest.

X-ray emission from PSR J1509-5850 can be reasonably well modeled with an absorbed power-law $\left(\chi_{v}=0.68\right.$ for 8 d.o.f.). This model yields a column density of $N_{\mathrm{H}}=$ $8.0_{-2.1}^{+2.3} \times 10^{21} \mathrm{~cm}^{-2}$, a photon index of $\Gamma=1.0_{-0.3}^{+0.2}$ and a normalization at $1 \mathrm{keV}$ of $5.1_{-1.6}^{+1.3} \times 10^{-6}$ photons $\mathrm{keV}^{-1} \mathrm{~cm}^{-2} \mathrm{~s}^{-1}$. The best-fitted model results in an unabsorbed flux of $f_{\mathrm{X}}=5.9 \times 10^{-14} \mathrm{erg} \mathrm{cm}^{-2} \mathrm{~s}^{-1}$ in the energy range of $0.5-8 \mathrm{keV}$. The dispersion-based distance implies a luminosity of $L_{\mathrm{X}}=$ $4.8 \times 10^{31}$ and $1.0 \times 10^{32} \mathrm{erg} \mathrm{s}^{-1}$ for $d=2.6$ and $3.8 \mathrm{kpc}$ respectively. Although a blackbody model can give a compatible goodness-of-fit $\left(\chi_{v}=0.82\right.$ for 8 d.o.f.), it infers a relatively high temperature $\left(T \sim 1.7 \times 10^{7} \mathrm{~K}\right)$ and a small projected blackbody radius $(R \sim 10 \mathrm{~m})$. Hence we regard this model as not physically reasonable to describe the X-ray spectrum of PSR J1509-5850. We note that the characteristic age indicates that PSR J1509-5850 belongs to the class of middle-aged pulsars. Their spectra typically consist of a soft thermal component, a harder thermal component from the heated polar caps as well as contribution from the non-thermal emission (Becker $\&$ Aschenbach 2002). However, the small number of collected

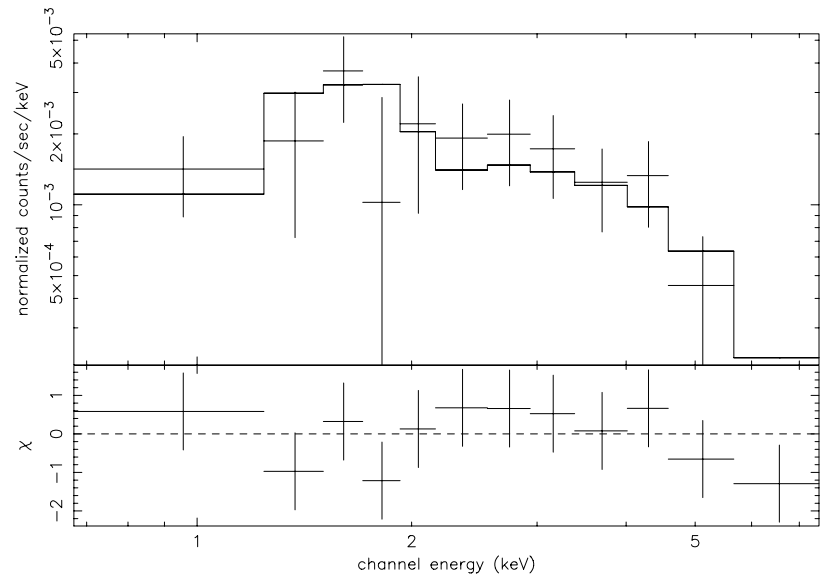

Fig. 2. Energy spectrum of the X-ray trail of PSR J1509-5850 as observed with the Chandra ACIS-S3 detector and fitted to an absorbed power-law model (upper panel) and contribution to the $\chi^{2}$ fit statistic (lower panel).

photons and the high column density does not support any fitting with multicomponent models.

We have tested the hypothesis that the trail emission originates from the interaction of pulsar wind and ISM by fitting an absorbed power-law model to the trail spectrum. The model yields an acceptable goodness-of-fit ( $\chi_{v}=0.73$ for 9 d.o.f.). The best fitting spectral model is displayed in Fig. 2. This model yields a column density of $N_{\mathrm{H}}=8.2_{-3.7}^{+9.3} \times 10^{21} \mathrm{~cm}^{-2}$, a photon index of $\Gamma=1.3_{-0.4}^{+0.8}$ and a normalization at $1 \mathrm{keV}$ of $1.9_{-1.9}^{+4.3} \times 10^{-5}$ photons $\mathrm{keV}^{-1} \mathrm{~cm}^{-2} \mathrm{~s}^{-1}$. We note that the column density agrees with that inferred from the pulsar spectrum. The unabsorbed flux deduced for the best-fitted model parameters are $f_{\mathrm{X}}=1.6 \times 10^{-13} \mathrm{erg} \mathrm{s}^{-1} \mathrm{~cm}^{-2}$ in the energy range of $0.5-8 \mathrm{keV}$. The dispersion-based distance implies a luminosity of $L_{\mathrm{X}}=1.3 \times 10^{32}$ and $2.7 \times 10^{32} \mathrm{erg} \mathrm{s}^{-1}$ for $d=2.6$ and $3.8 \mathrm{kpc}$, respectively.

We searched for a possible radio counterpart for the X-ray nebula with the Sydney University Molonglo Sky Survey data (SUMSS) (Bock et al. 1999) and discovered a long radio trail apparently associated with PSR J1509-5850. The radio image of the $11 \times 11$ arcmin field near to PSR J1509-5850 is displayed in Fig. 3. The radio feature has a length of $\sim 7$ arcmin. Radio contours were calculated at the levels of 7, 23, 39, 54 and $70 \mathrm{mJy} / \mathrm{beam}$. These contours were overlaid on the image of the Chandra ACIS-S3 chip in Fig. 4. It is interesting to note that the radio trail starts exactly from the position of PSR J1509-5850 and has the same orientation as that of the X-ray trail. This supports the interpetation that this extended radio feature is the radio counterpart of the X-ray trail and is indeed physically associated with PSR J1509-5850.

Two clumpy structures are observed along the radio trail (see Fig. 3). The northern clump has its emission center at $\mathrm{RA}=15^{\mathrm{h}} 09^{\mathrm{m}} 14.35^{\mathrm{s}}$, Dec $=-58^{\circ} 54^{\prime} 50.7^{\prime \prime}(\mathrm{J} 2000)$ with a radius of $\sim 1.5 \mathrm{arcmin}$. The southern clump has its emission center at RA $=15^{\mathrm{h}} 09^{\mathrm{m}} 06.33^{\mathrm{s}}$, Dec $=-58^{\circ} 58^{\prime} 34.7^{\prime \prime}(\mathrm{J} 2000)$ with a radius of $\sim 1$ arcmin. While the southern clump is unidentified in SIMBAD and NED, the northern clump, which is located $\sim 4$ arcmin away from PSR J1509-5850, has been proposed to be a supernova remnant candidate MSC 319.9-0.7 (Whiteoak \& Green 1996). Comparing the X-ray and the radio data in Fig. 4 , we found some faint X-ray emission near to the location of MSC 319.9-0.7. The emission does not appear to be the continuation 


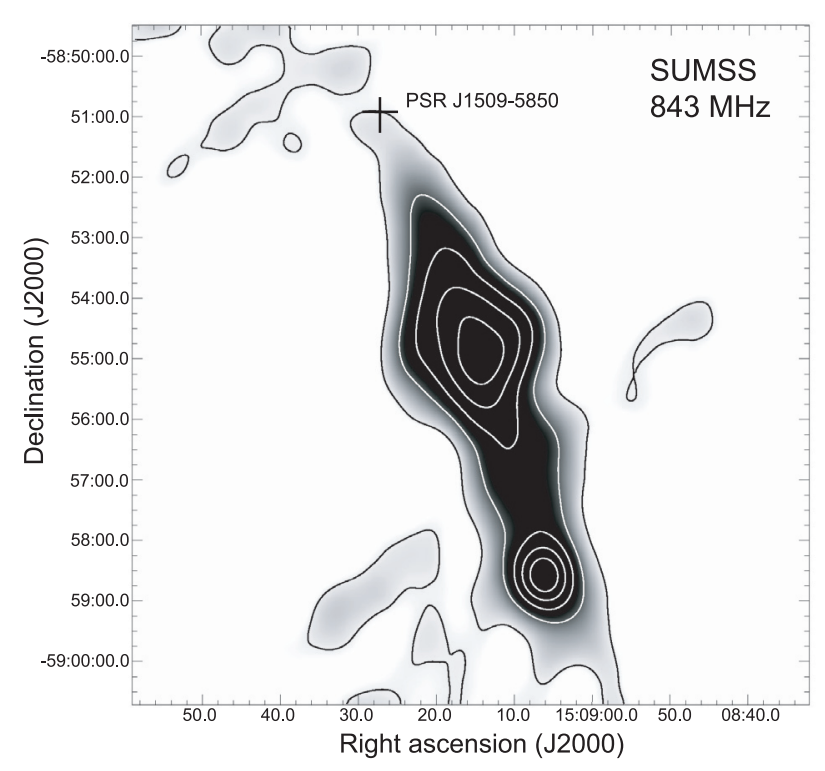

Fig. 3. The $843 \mathrm{MHz}$ SUMSS image of a field of $11 \times 11$ arcmin around PSR J1509-5850. The pulsar position is indicated by the black cross. The $\sim 7$ arcmin long radio feature has the same orientation as the X-ray trail. The contour levels are 7, 23, 39, 54 and $70 \mathrm{mJy} /$ beam. Two clumps are observed along the trail. The larger clump, near to the center of this image, on the trail is the SNR candidate MSC 319.9-0.7.

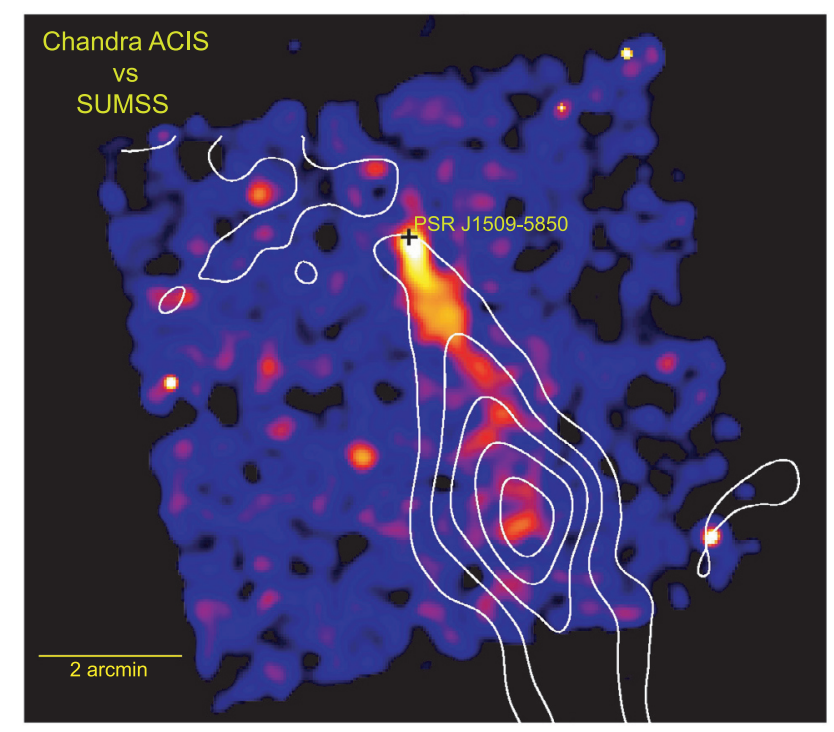

Fig. 4. The X-ray image of the Chandra ACIS-S3 chip with the radio contour lines from SUMSS data (Fig. 3) overlaid. The X-ray image is binned with a pixel size of 2.5 arcsec and adaptively smoothed with a Gaussian kernel of $\sigma<7.5$ arcsec. We note a faint X-ray feature near the location of the SNR candidate MSC 319.9-0.7. Top is north and left is east.

of the trail associated with PSR J1509-5850. It cannot be excluded that this faint emission is related to MSC 319.9-0.7. However, the limited photon statistics does not allow final conclusion to be drawn.

\section{Discussion and conclusion}

In this paper, we report the detection of a possible radio counterpart of the X-ray trail associated with PSR J1509-5850 and present a first detailed X-ray study of the X-ray trail. Apart from the radio trail, we found two clumpy structures located on the trail. While the smaller one is unidentified, the larger one, which is located $\sim 4$ arcmin away from PSR J1509-5850, is a SNR candidate MSC 319.9-0.7.

Despite the proximity of MSC 319.9-0.7, it seems unlikely that it is the birth place of PSR J1509-5850. Assuming this shell-like SNR candidate is in a Sedov phase, the radius of the shocked shell emission can be estimated by (Culhane 1997):

$R_{\mathrm{s}}=2.15 \times 10^{-11}\left(\frac{E}{n}\right)^{\frac{1}{5}} t^{\frac{2}{5}} \mathrm{pc}$

where $t, E$ and $n$ are the time after the explosion in units of years, the released kinetic energy in units of erg and the ISM number density in units of $\mathrm{cm}^{-3}$ respectively. Taking the typical values of $E=10^{51} \mathrm{erg}$ and $n=1 \mathrm{~cm}^{-3}$ and $t$ to be the characteristic age of PSR J1509-5850, we estimate that a SNR associated with PSR J1509-5850 should have a radius of $R_{\mathrm{S}} \sim 40 \mathrm{pc}$. However, MSC 319.9-0.7 only has a radius of $\sim 1.1-1.7$ pc for $d=2.6-$ $3.8 \mathrm{kpc}$. Thus, the discrepancy between the expected $R_{\mathrm{S}}$ and the observed value with a factor of $\sim 30$ is unlikely to be reconciled by the uncertainty of the dispersion based distance. On the other hand, the characteristic age of the pulsar can be older than its actual age if its inital spin period was close to its current period. However, to reconcile such discrepancy would require $t$ to be smaller by a factor of $\sim 4000$ which is not likely. Moreover, associating MSC 319.9-0.7 with PSR J1509-5850 would leave the origin of the southern part of the radio trail unexplained. Thus, with the current knowledge of parameters it seems most reasonable to interpret MSC 319.9-0.7 as a background source.

Following the discussion in Hui \& Becker (2006), we apply a simple one-zone model (Chevalier 2000; Cheng et al. 2004) to model the X-ray emission properties of the pulsar wind nebula. Since the proper motion of PSR J1509-5850 is not yet known, we assume the pulsar is in supersonic motion on the basis that the nebula resembles a bow-shock morphology. For the supersonic motion, the termination shock radius $R_{\mathrm{ts}}$ is determined by the balance of the ram pressure between the relativistic pulsar wind particles and the ISM at the head of the shock (Cheng et al. 2004):

$R_{\mathrm{ts}} \simeq\left(\frac{\dot{E}}{2 \pi \rho_{I S M} v_{\mathrm{p}}^{2} c}\right)^{1 / 2} \sim 3 \times 10^{16} \dot{E}_{34}^{1 / 2} n^{-1 / 2} v_{\mathrm{p}, 100}^{-1} \mathrm{~cm}$

where $v_{\mathrm{p}, 100}$ is the velocity of the pulsar in units of $100 \mathrm{~km} \mathrm{~s}^{-1}$, $\dot{E}_{34}$ is the spin-down luminosity of the pulsar in units of $10^{34} \mathrm{erg} \mathrm{s}^{-1}$, and $n$ is the number density of the ISM in units of $\mathrm{cm}^{-3}$. In all following estimations, we assume PSR J1509-5850 has a transverse velocity comparable to the average velocity, $\sim 250 \mathrm{~km} \mathrm{~s}^{-1}$, of ordinary radio pulsars (Hobbs et al. 2005). For a ISM density of $1 \mathrm{~cm}^{-3}$, Eq. (2) implies a termination radius of $R_{\mathrm{ts}} \sim 8.6 \times 10^{16} \mathrm{~cm}$.

The X-ray trail is found to be $\sim 2$ arcmin long. For the dispersion-based distance in the range of $\sim 2.6-3.8 \mathrm{kpc}$, the trail has a length of $l \sim(4.7-6.8) \times 10^{18} \mathrm{~cm}$. For the assumed pulsar velocity of $\sim 250 \mathrm{~km} \mathrm{~s}^{-1}$, the timescale for the passage of the pulsar over the length of its X-ray trail, $t_{\text {flow }}$, is estimated to be $\sim 6000-8600$ years. The magnetic field in the shocked region can be estimated by assuming $t_{\text {flow }}$ to be comparable to the synchrotron cooling timescale of electrons:

$\tau_{\mathrm{syn}}=\frac{6 \pi m_{\mathrm{e}} c}{\gamma \sigma_{\mathrm{T}} B^{2}} \simeq 10^{5}\left(\frac{h v}{\mathrm{keV}}\right)^{-\frac{1}{2}} B_{\mu \mathrm{G}}^{-\frac{3}{2}} \mathrm{yr}$ 
where $\gamma$ is the Lorentz factor of the wind, taken to be $10^{6}$ (Cheng et al. 2004), $\sigma_{\mathrm{T}}$ is the Thompson cross section, and $B_{\mu \mathrm{G}}$ is the magnetic field in the shocked region in units of micro gauss. The inferred magnetic field in the shocked region is $\sim 5-7 \mu \mathrm{G}$. For comparison, the magnetic field strength in the ISM is estimated to be $\sim 2-6 \mu \mathrm{G}$ (Beck et al. 2003, and references therein).

The X-ray luminosity and spectral index depend on the inequality between the characteristic observed frequency $v_{\mathrm{X}}^{\mathrm{obs}}$ and the electron synchrotron cooling frequency $v_{\mathrm{c}}$ (see Chevalier 2000, and references therein):

$\nu_{\mathrm{c}}=\frac{18 \pi e m_{\mathrm{e}} c}{\sigma_{\mathrm{T}}^{2} \tau_{\mathrm{syn}}^{2} B^{3}}$

which is estimated to be $v_{\mathrm{c}}=(1.3-1.8) \times 10^{17} \mathrm{~Hz}$. Since in general $v_{\mathrm{X}}^{\mathrm{obs}}>v_{\mathrm{c}}$, this suggests the X-ray emission is in a fast cooling regime. Electrons with the energy distribution, $N(\gamma) \propto \gamma^{-p}$, are able to radiate their energy in the trail with photon index $\alpha=(p+2) / 2$. The index $p$ due to shock acceleration typically lies between 2 and 3 (cf. Cheng et al. 2004, and reference therein). This would result in a photon index $\alpha \simeq 2.0-2.5$. In view of the large error of the observed photon index $\Gamma=1.3_{-0.4}^{+0.8}$, we cannot firmly conclude the emission scenario simply based on the photon index. We note that the photon index can still be possibly in the fast cooling regime within the $1 \sigma$ uncertainty. With this consideration and $v_{\mathrm{X}}^{\mathrm{obs}}>v_{\mathrm{c}}$, we adopted the fast cooling scenario in the following discussion. With the assumed value $p=2.2$, the calculated photon index $\alpha=2.1$ which is marginally within the $1 \sigma$ uncertainty of the observed value. In a fast cooling regime, the luminosity per unit frequency is given by (Cheng et al. 2004):

$L_{v}=\frac{1}{2}\left(\frac{p-2}{p-1}\right)^{p-1}\left(\frac{6 e^{2}}{4 \pi^{2} m_{\mathrm{e}} c^{3}}\right)^{\frac{p-2}{4}} \epsilon_{\mathrm{e}}^{p-1} \epsilon_{\mathrm{B}}^{\frac{p-2}{4}} \gamma^{p-2} R_{\mathrm{ts}}^{-\frac{p-2}{2}} \dot{E}^{\frac{p+2}{4}} v^{-\frac{p}{2}}$.

Assuming the energy equipartion between the electron and proton, we take the fractional energy density of electron $\epsilon_{\mathrm{e}}$ to be $\sim 0.5$ and the fractional energy density of the magnetic field $\epsilon_{\mathrm{B}}$ to be $\sim 0.01$. We integrate Eq. (5) from $0.5 \mathrm{keV}$ to $8 \mathrm{keV}$, resulting in a calculated luminosity of $\sim 6 \times 10^{32} \mathrm{erg} \mathrm{s}^{-1}$. With the reasonable choice of parameters stated above, the luminosity estimated by this simple model is found to be the same order as the observed value.

It is obvious that the radio nebula is significantly longer than its X-ray counterpart (Fig. 4). This is not unexpected. Considering a scenario of constant injection of particles with a finite synchrotron cooling time, the number of particles that can reach to a further distance from the pulsar should decrease with increasing frequency. This is because the synchrotron cooling timescale decreases with frequency. This results in synchrotron nebular size decreasing with frequency.

To further constrain the physical properties of the pulsar wind nebula associated with PSR J1509-5850, multi-wavelength observations are essential. Since SUMSS data have a rather poor spatial resolution which has a typical beam size of $\sim 45$ arcsec, details of the nebular emission might be remain unresolved. In particular, it is important to better resolve the nebular emission from the contribution of the SNR candidate MSC 319.9-0.7. In view of this, high resolution radio observations (e.g. ATCA) are required. In the X-ray regime, although the Chandra observation has already provided us with a high resolution image of the nebula, the photon statistics are not sufficient to tightly constrain the spectral properties. Owing to the superior collecting power, observations with XMM-Newton are expected to put a tight constraint on the emission nature of the nebula as well as the pulsar itself.

Apart from the radio and X-ray observations, a complete study of pulsar wind nebula should also include TeV observations (e.g. HESS). It is generally believed that the $\mathrm{TeV}$ photons result from inverse Compton scattering of soft photon field by the relativistic particles in the nebulae. The seed soft photons are possibly contributed by the cosmic microwave background (Cui 2006). However, only a handful of pulsar wind nebulae have been detected in TeV regime so far (Cui 2006). A larger sample is needed for differentiating the aforementioned interpetation from its competing scenario (e.g. neutral pion decay).

From the above discussion, one should note that the pulsar's transverse velocity is an important parameter in studying shock physics. Hence measuring the proper motion of PSR J1509-5850 is essential. Moreover, although the orientation of the trail suggests PSR J1509-5850 is probably moving northeast, it is not necessary for the trail to be aligned with the pulsar velocity. PSRs J2124-3358 and B2224+65 are examples that the X-ray trails are misaligned with the direction of the pulsars' proper motion (Hui \& Becker 2006, 2007).

\section{References}

Becker, W., Aschenbach, B. 2002, in Neutron Stars, Pulsars and Supernova Remnants, ed. W. Becker, H. Lesch \& J. Trümper, MPE Rep., 278, 64, Beck, R., Shukurov, A., Sokoloff, D., \& Wielebinski, R. 2003, A\&A, 411, 99 Bock, D. C.-J., Large, M. I., \& Sadler, Elaine M. 1999, AJ, 117, 1578

Cheng, K. S., Taam, R. E., \& Wang, W. 2004, ApJ, 617, 480 Chevalier, R. A. 2000, ApJ, 539, L45

Cordes, J. M., \& Lazio, T. J. W. 2002, [arXiv: astro-ph/0207156]

Cui, W. 2006, [arXiv: astro-ph/0608042]

Culhane, J. L. 1977, in Supernovae, ed., D. N. Schram (Dordrecht, Holland: D. Reidel Publishing Co.) 29

Hobbs, G., Lorimer, D. R., Lyne, A. G., \& Kramer, M. 2005, MNRAS, 360, 974 Hui, C. Y., \& Becker, W. 2006, A\&A, 448, L13

Hui, C. Y., \& Becker, W. 2007, A\&A, 467, 1209

Kargaltsev, O., et al. 2006, A\&AS HEAD meeting 9

Manchester, R. N., Lyne, A. G., Camilo, F., et al. 2001, MNRAS, 328, 17

Manchester, R. N., Hobbs, G. B., Teoh, A., \& Hobbs, M. 2005, AJ, 129, 1993

Monet, D. B. A., Canzian, B., Dahn, C., et al. 1998, VizieR Online Data Catalog, 1252,0

Taylor, J. H., \& Cordes, J. M 1993, ApJ, 411, 674

Whiteoak, J. B. Z., \& Green, A. J. 1996, A\&AS, 118, 329 